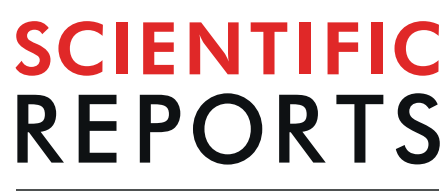

\title{
Etiologic features of diarrheagenic microbes in stool specimens from patients with acute diarrhea in Thailand
}

\author{
Kazuhisa Okada $\mathbb{1}^{1,2^{*}}$, Warawan Wongboot ${ }^{1,3}$, Watcharaporn Kamjumphol ${ }^{1}$, \\ Namfon Suebwongsa ${ }^{1}$, Piyada Wangroongsarb ${ }^{3}$, Pipat Kluabwang ${ }^{4}$, Nuttagarn Chuenchom ${ }^{5}$, \\ Witaya Swaddiwudhipong ${ }^{6}$, Thanee Wongchai ${ }^{7}$, Weerawat Manosuthi ${ }^{8}$, \\ Norrathep Assawapatchara ${ }^{9}$, Patchanee Khum-on ${ }^{10}$, Patpong Udompat ${ }^{11}$, \\ Chareeya Thanee ${ }^{12}$, Suwatthiya Kitsaran ${ }^{13}$, Lakkana Jirapong ${ }^{14}$, Charoen Jaiwong ${ }^{15}$, \\ Supalert Nedsuwan ${ }^{16}$, Chotipong Siripipattanamongkol ${ }^{17}$, Pilailuk Akkapaiboon Okada ${ }^{3}$, \\ Siriporn Chantaroj ${ }^{3}$, Sho Komukai ${ }^{18}{ }^{18}$ \& Shigeyuki Hamada ${ }^{1,2}$
}

Many microbial species have been recognized as enteropathogens for humans. Here, we predicted the causative agents of acute diarrhea using data from multiplex quantitative PCR (qPCR) assays targeting 19 enteropathogens. For this, a case-control study was conducted at eight hospitals in Thailand. Stool samples and clinical data were collected from 370 hospitalized patients with acute diarrhea and 370 non-diarrheal controls. Multiple enteropathogens were detected in $75.7 \%$ and $13.0 \%$ of diarrheal stool samples using multiplex qPCR and bacterial culture methods, respectively. Asymptomatic carriers of enteropathogens were found among $\mathbf{8 7 . 8 \%}$ and $\mathbf{4 5 . 7 \%}$ of individuals by qPCR and culture methods, respectively. These results suggested the complexity of identifying causative agents of diarrhea. An analysis using the quantification cut-off values for clinical relevance drastically reduced pathogenpositive stool samples in control subjects from $87.8 \%$ to $0.5 \%$, whereas $48.9 \%$ of the diarrheal stool samples were positive for any of the 11 pathogens. Among others, rotavirus, norovirus GII, Shigellal EIEC, and Campylobacter were strongly associated with acute diarrhea $(P$-value $<0.001)$. Characteristic clinical symptoms, epidemic periods, and age-related susceptibility to infection were observed for some enteropathogens. Investigations based on qPCR approaches covering a broad array of enteropathogens might thus improve our understanding of diarrheal disease etiology and epidemiological trends.

Diarrheal diseases are one of the major causes of mortality and morbidity worldwide, especially during the first 5 years of life for individuals subjected to malnutrition ${ }^{1-3}$. Diarrhea can be defined by increased stool frequency, liquidity, or volume ${ }^{4}$. A wide range of enteropathogens including bacteria, viruses, and protozoa have been

\footnotetext{
${ }^{1}$ Thailand-Japan Research Collaboration Center on Emerging and Re-emerging Infections, Nonthaburi, Thailand. ${ }^{2}$ Research Institute for Microbial Diseases, Osaka University, Osaka, Japan. ${ }^{3}$ National Institute of Health, Department of Medical Sciences, Nonthaburi, Thailand. ${ }^{4}$ Department of Pediatrics, Maesot General Hospital, Tak, Thailand. ${ }^{5}$ Department of Medicine, Maesot General Hospital, Tak, Thailand. ${ }^{6}$ Department of Community and Social Medicine, Maesot General Hospital, Tak, Thailand. ${ }^{7}$ Department of Clinical Laboratory, Maesot General Hospital, Tak, Thailand. ${ }^{8}$ Department of Medicine, Bamrasnaradura Infectious Diseases Institute, Nonthaburi, Thailand. ${ }^{9}$ Department of Medicine, Ranong Hospital, Ranong, Thailand. ${ }^{10}$ Department of Medical Technology, Chum Phae Hospital, Khon Kaen, Thailand. ${ }^{11}$ Department of Community and Social Medicine, Prapokklao Hospital, Chanthaburi, Thailand. ${ }^{12}$ Department of Pediatrics, Sunpasitthiprasong Hospital, Ubon Ratchathani, Thailand. ${ }^{13}$ Department of Medicine, Sunpasitthiprasong Hospital, Ubon Ratchathani, Thailand. ${ }^{14}$ Department of Radiology, Samutsakhon Hospital, Samutsakhon, Thailand. ${ }^{15}$ Department of Pediatrics, Chiangrai Prachanukroh Hospital, Chiang Rai, Thailand. ${ }^{16}$ Department of Preventive and Social Medicine, Chiangrai Prachanukroh Hospital, Chiang Rai, Thailand. ${ }^{17}$ Department of Medicine, Chiangrai Prachanukroh Hospital, Chiang Rai, Thailand. ${ }^{18}$ Department of Integrated Medicine of Graduate School of Medicine, Osaka University, Osaka, Japan. *email: kazuhisa@biken.osaka-u.ac.jp
} 

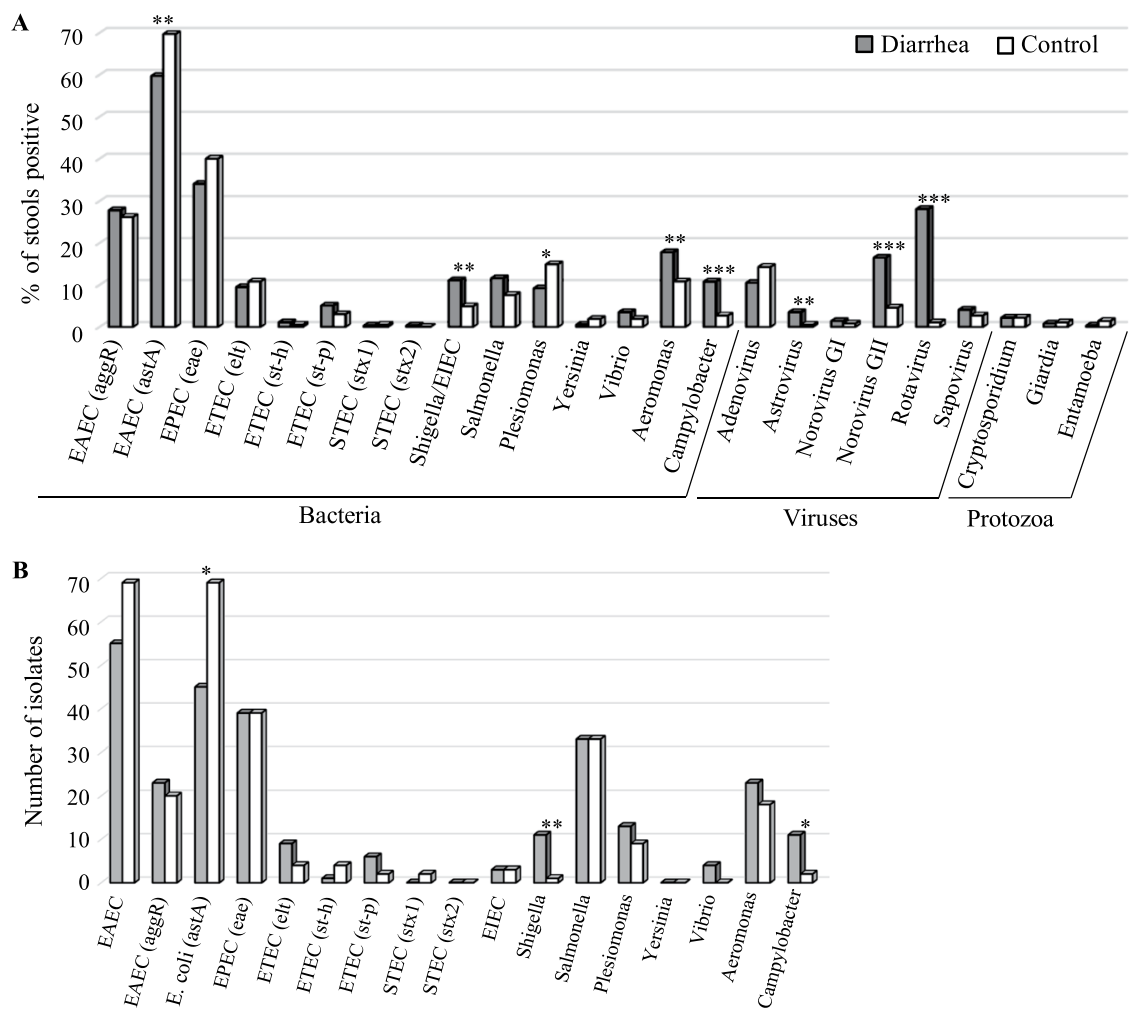

Figure 1. Detection of enteropathogens in 370 diarrheal and 370 non-diarrheal control subjects. (A) Proportions of patients and control subjects who tested positive by qPCR assays. (B) Number of isolates by bacterial culture methods. $* P<0.05$; ** $P<0.01$; *** $P<0.001$.

recognized as the causative agents of infectious diarrhea ${ }^{5,6}$. Several enteropathogens act either directly by modulating epithelial ion transport systems and barrier functions or indirectly via neuropeptide secretion, induction of inflammation, or by compromising intestinal absorption ${ }^{7}$. Thus, the timely identification of causes of acute diarrhea can lead to appropriate treatment, prevention, and control measures. Molecular assays targeting nucleic acid markers facilitate the screening of a broad range of enteropathogens in stool samples, consuming much less time than conventional methods such as cell culture, microscopy, and antigen-based tests. However, due to the high sensitivity of these nucleic acid amplification assays, high rates of asymptomatic carriers and mixed infection cases are commonly reported, particularly in developing country settings ${ }^{8-12}$. Several studies have shown correlations between pathogen load and severity, or have accurately diagnosed individual pathogens using qPCR assays with optimal cut-off values ${ }^{13-21}$.

In this study, we predicted the etiological agents of acute diarrhea using stool samples from hospitalized patients in Thailand using our quantitative pathogen detection procedure ${ }^{22}$ with clinically relevant cut-off values. We further investigated characteristic clinical symptoms, epidemic periods, and age-related susceptibility to infection associated with the detected enteropathogens.

\section{Results}

Detection of diarrheagenic microbes in stool specimens using multiplex qPCR assays. Stool specimens (370 each) from cases and controls were investigated using a multiplex qPCR panel assay to detect 24 target genes (Fig. 1A). The pathogen detection rates of bacteria, viruses, and parasites in diarrhea cases were $84.9 \%, 49.2 \%$, and $3.2 \%$, whereas the corresponding rates in the controls were $83.8 \%, 20.8 \%$, and $4.9 \%$, respectively. The numbers of Escherichia coli including enteroaggregative E. coli (EAEC) (aggR, astA) and enteropathogenic E. coli (EPEC) (eae) were relatively high in both cases and controls. Shigella/EIEC (odds ratio (OR) 2.40, $P=0.003$ ), Aeromonas (OR 1.78, $P=0.008$ ), Campylobacter (OR 4.21, $P<0.001$ ), astrovirus (OR 5.57, $P=0.007$ ), norovirus GII (OR 4.01, $P<0.001$ ), and rotavirus (OR 31.94, $P<0.001$ ) were detected more often in patients than in controls (Table 1). The detection rates of enteric adenovirus in the adenovirus-positive samples assessed using other qPCR assays ${ }^{23}$ were also not significant (OR 1.3, $P=0.393$ ) (data not shown). EAEC (ast A) (OR $0.64, P=0.004$ ) and Plesiomonas (OR $0.55, P=0.013$ ) were more common in non-diarrhea controls without an adjusted Cq cut-off value. An average of $2.7 \pm 0.1$ (standard error) pathogens was detected using qPCR in patients with diarrhea, whereas $2.3 \pm 0.1$ were detected in the controls (Fig. 2A). The percentages of "no pathogen detected" and mixed infection were $5.9 \%$ and $75.7 \%$ (IQR, 3; mean, 3.3) in cases, and $12.2 \%$ and 66.5\% (IQR, 2; mean, 3.1 ) in controls, respectively. 
A

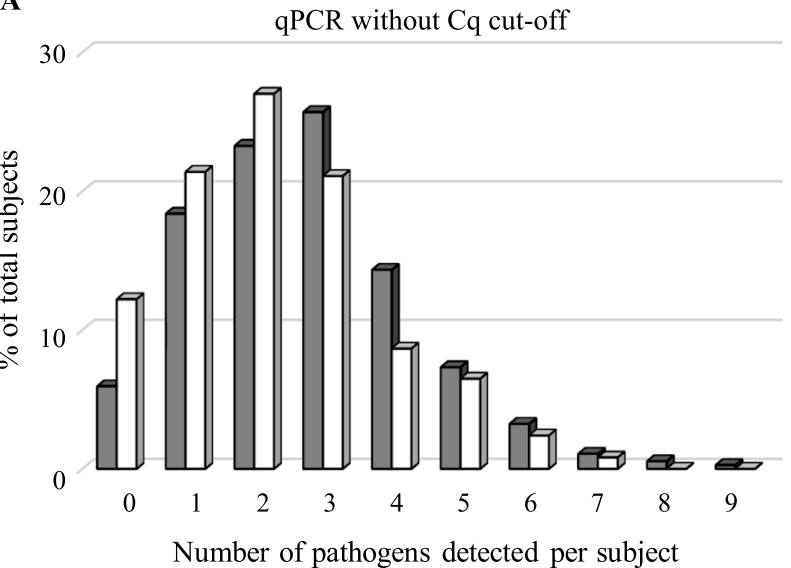

B

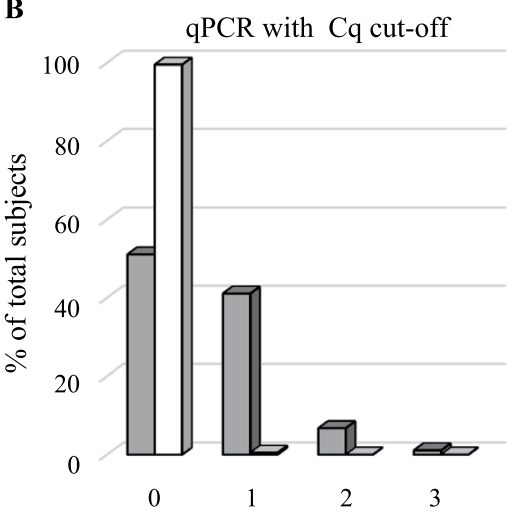

Number of pathogens detected per subject

$\square$ Diarrhea $\square$ Control

Figure 2. Number of pathogens detected per subject using qPCR among 370 each of diarrheal (an average of $2.7 \pm 0.1$ (standard error) pathogens) and non-diarrheal control subjects (an average of $2.3 \pm 0.1$ (standard error) pathogens) (A) and the distribution based on the quantitative cycle (Cq) cut-off values mentioned in Table $1(\mathbf{B})$.

\begin{tabular}{|c|c|c|c|c|c|c|c|c|c|}
\hline & \multicolumn{4}{|c|}{ Detection by quantitative PCR } & \multirow{2}{*}{$\begin{array}{l}\text { Cq cutoff } \\
\text { value }\end{array}$} & \multicolumn{4}{|c|}{ Detection based on optimal cut-off } \\
\hline & Diarrhea (\%) & Control (\%) & OR & $P$-value & & Diarrhea (\%) & Control (\%) & OR & $P$-value \\
\hline Rotavirus & $104(28.1)$ & $4(1.1)$ & 31.94 & $<0.001$ & 27.98 & $80(21.6)$ & $0(0)$ & 205.34 & $<0.001$ \\
\hline Norovirus GII & $61(16.5)$ & $17(4.6)$ & 4.01 & $<0.001$ & 20.23 & $29(7.8)$ & $0(0)$ & 64.01 & $<0.001$ \\
\hline Shigella/EIEC & $41(11.1)$ & $18(4.9)$ & 2.40 & 0.003 & 30.06 & $21(5.7)$ & $0(0)$ & 45.58 & $<0.001$ \\
\hline Campylobacter & $40(10.8)$ & $10(2.7)$ & 4.21 & $<0.001$ & 28.97 & $18(4.9)$ & $0(0)$ & 38.89 & $<0.001$ \\
\hline EAEC (aggR) & $103(27.8)$ & $99(26.8)$ & 1.06 & 0.805 & 22.54 & $11(3.0)$ & $0(0)$ & 23.70 & 0.001 \\
\hline EAEC (astA) & $221(59.7)$ & $259(70.0)$ & 0.64 & 0.004 & 19.95 & $10(2.7)$ & $1(0.3)$ & 7.17 & 0.011 \\
\hline Aeromonas & $66(17.8)$ & $40(10.8)$ & 1.78 & 0.008 & 27.42 & $9(2.4)$ & $0(0)$ & 19.47 & 0.004 \\
\hline Sapovirus & $15(4.1)$ & $10(2.7)$ & 1.50 & 0.416 & 27.20 & $9(2.4)$ & $0(0)$ & 19.47 & 0.004 \\
\hline Astrovirus & $13(3.5)$ & $2(0.5)$ & 5.57 & 0.007 & 26.21 & $8(2.2)$ & $1(0.3)$ & 5.78 & 0.038 \\
\hline Salmonella & $43(11.6)$ & $28(7.6)$ & 1.60 & 0.080 & 25.56 & $7(1.9)$ & $0(0)$ & 15.29 & 0.015 \\
\hline Plesiomonas & $33(8.9)$ & $56(15.1)$ & 0.55 & 0.013 & 26.17 & $6(1.6)$ & $0(0)$ & 13.21 & 0.031 \\
\hline \multirow[t]{3}{*}{ ETEC (elt) } & $35(9.5)$ & $40(10.8)$ & 0.86 & 0.626 & 25.29 & $6(1.6)$ & $0(0)$ & 13.21 & 0.031 \\
\hline & \multicolumn{4}{|c|}{ Detection by quantitative PCR } & \multirow{2}{*}{$\begin{array}{l}\text { Cq cutoff } \\
\text { value }\end{array}$} & \multicolumn{4}{|c|}{ Detection based on optimal cut-off } \\
\hline & Diarrhea (\%) & Control (\%) & OR & $P$-value & & Diarrhea (\%) & Control (\%) & OR & $P$-value \\
\hline EPEC & $125(34.1)$ & $149(40.3)$ & 0.77 & 0.094 & 21.08 & $5(1.4)$ & $0(0)$ & 11.15 & 0.062 \\
\hline $\operatorname{ETEC}(s t-p)$ & $19(5.1)$ & $11(3.0)$ & 1.73 & 0.191 & 26.24 & $4(1.1)$ & $0(0)$ & 9.10 & 0.124 \\
\hline Adenovirus & $39(10.5)$ & $53(14.3)$ & 0.71 & 0.147 & 14.45 & $4(1.1)$ & $0(0)$ & 9.10 & 0.124 \\
\hline Vibrio & $13(3.5)$ & $8(2.2)$ & 1.61 & 0.376 & 30.09 & $3(0.8)$ & $0(0)$ & 7.06 & 0.249 \\
\hline Norovirus GI & $5(1.4)$ & $3(0.8)$ & 1.58 & 0.725 & 28.02 & $3(0.8)$ & $0(0)$ & 7.06 & 0.249 \\
\hline $\operatorname{ETEC}(s t-h)$ & $4(1.1)$ & $2(0.5)$ & 1.81 & 0.686 & - & $0(0)$ & $0(0)$ & 1 & 1 \\
\hline Cryptosporidium & $8(2.2)$ & $8(2.2)$ & 1 & 1 & - & $0(0)$ & $0(0)$ & 1 & 1 \\
\hline Giardia & $3(0.8)$ & $4(1.1)$ & 0.78 & 1 & 24.52 & $1(0.3)$ & $0(0)$ & 3.01 & 1 \\
\hline Entamoeba & $1(0.3)$ & $6(1.6)$ & 0.23 & 0.123 & - & $0(0)$ & $0(0)$ & 1 & 1 \\
\hline Yersinia & $2(0.5)$ & $7(1.9)$ & 0.33 & 0.177 & - & $0(0)$ & $0(0)$ & 1 & 1 \\
\hline STEC (stx1) & $1(0.3)$ & $2(0.5)$ & 0.60 & 1 & - & $0(0)$ & $0(0)$ & 1 & 1 \\
\hline STEC (stx2) & $1(0.3)$ & $0(0)$ & 3.01 & 1 & 37.74 & $1(0.3)$ & $0(0)$ & 3.01 & 1 \\
\hline
\end{tabular}

Table 1. Detection of enteropathogen targets in stool samples from cases and control subjects, odds ratio, Cq cutoff value, and predicted causative agents by quantitative PCR analyses. The 12 targeted genes in 11 enteropathogens in the top half of the table were determined as the causative agents of acute diarrhea. Receiver operating curve (ROC) analyses were conducted to detect the optimal cut-off value. The cut-off value with the largest OR, which satisfied more than $95 \%$ specificity, was considered optimal. The relationships between case (control) and positive or negative control based on the calculated cut-off value were reported as ORs and P-values determined using the Fisher's exact test. Abbreviations: OR, odd ratio; Cq, quantification cycle; EIEC, Enteroinvasive E. coli; EAEC, Enteroaggregative E. coli; ETEC, Enterotoxigenic E. coli; EPEC, Enteropathogenic E. coli; STEC, Shiga toxin-producing E. coli. 
Cultivation and identification of bacterial enteropathogens isolated from stool specimens. Two hundred and thirty-four and 230 bacterial pathogens consisting of Aeromonas caviae, A. hydrophila, A. sobria, A. veronii, Campylobacter coli, C. fetus, C. jejuni, E. coli, Plesiomonas shigelloides, Salmonella enterica, Shigella boydii, S. flexneri, S. sonnei, Vibrio fluvialis, and V. parahaemolyticus were isolated from 170 cases (45.9\%) and 169 controls (45.7\%), respectively (Fig. 1B, Table 2). Multiple bacterial pathogens were isolated from $13.0 \%$ cases and $12.7 \%$ controls (Table 3). The most common bacteria were E. coli (31.4\% vs $37.0 \%$ ), Salmonella (8.9\% vs $8.9 \%$ ), and Aeromonas (5.9\% vs 4.3\%) in both cases and controls (Table 2). The isolation rates of Shigella and Campylobacter species were statistically associated with diarrhea, and S. sonnei and C. jejuni were the dominant species. Further, 306 isolates of $E$. coli were classified into 44 genotypes by multiplex-PCR assays and $\geq 8$ different O-serotypes were identified among 66 Salmonella isolates. Most bacterial pathogens were similarly distributed in both patients and healthy control subjects.

Statistical analysis of qPCR data with the clinically relevant cut-off values. Due to the issues associated with high rates of asymptomatic carriage and the detection of multiple pathogens in patients, we next compared the quantification cycle values $(\mathrm{Cq})$ of $\mathrm{qPCR}$ for each target gene in the case and control samples (Fig. S1). Cq cut-off values for each pathogen were determined using receiver operating curve (ROC) analysis (see Methods). Results showed that 12 targets had statistically significant associations with diarrhea occurrence (Table 1). With the clinically relevant cut-off values, pathogen-positive stool samples in control subjects were drastically reduced from $87.8 \%$ to $0.5 \%$, whereas 181 diarrheal stools ( $48.9 \%$ ) were positive for any of the 11 enteropathogens - rotavirus, norovirus GII, Shigella/enteroinvasive E. coli (EIEC), Campylobacter, EAEC, Salmonella, Plesiomonas, Aeromonas, sapovirus, astrovirus, and enterotoxigenic E. coli (ETEC) - that were strongly associated with acute diarrhea (Fig. 2B, Table 1). Moreover, rotavirus, norovirus GII, Shigella/enteroinvasive E. coli (EIEC), Campylobacter, EAEC, Salmonella, Plesiomonas, Aeromonas, sapovirus, astrovirus, and enterotoxigenic E. coli (ETEC) were strongly associated with acute diarrhea. Among 181 patients, single and multiple infections with the causative agents accounted for $84.0 \%$ and $16.0 \%$, cases, respectively (Table 4). Mixed infection cases including bacterial-viral $(7.7 \%, 14 / 181)$, bacterial-bacterial $(4.4 \%, 8 / 181)$, and viral-viral $(3.9 \%, 7 / 181)$ infections were still recognized despite the use of the stringent cut-off values. These mixed infection cases were observed more frequently in younger age groups ( $\leq 15$ years of age) than in older age groups $(\geq 16$ years old $)(P=0.037)$.

Patient features and clinical symptoms that might be caused by the detected pathogens. Patient features and clinical symptoms that might be caused by the detected pathogens were then investigated. Patients with a high frequency of defecation (more than 10 times per day) had significantly higher numbers of Aeromonas than all cases $(P=0.006)$ (Table $S 1)$. Rotavirus infection was significantly associated with fever $(P=0.034)$. Moreover, bacterial infections rather than viral infections tended to cause abdominal pain $(P<0.001)$. Shigella/EIEC $(P=0.020)$, Salmonella $(P=0.013)$, Aeromonas $(P=0.027)$, and Plesiomonas $(P=0.037)$ were more related to abdominal pain, whereas norovirus GII infection was rare in individuals with abdominal pain $(P=0.014)$. More than $20 \mathrm{WBCs} / \mathrm{hpf}$ (white blood cells per high power field) and red blood cells were present in the stool samples of patients with Shigella/EIEC infection $(P<0.001$ and $P=0.012$, respectively). Vomiting frequency was higher in rotavirus- $(P<0.001)$ and norovirus GII -infected patients $(P=0.009)$ than in all patients, but the incidence of nausea was significantly associated with Aeromonas- $(P=0.020)$ and sapovirus-infected patients $(P=0.037)$. Rotavirus $(P=0.001)$, norovirus GII $(P=0.004)$, and Campylobacter $(P=0.040)$ were more often detected in patients less than five years of age, whereas Shigella/EIEC $(P=0.001)$, Aeromonas $(P=0.010)$, and Plesiomonas $(P=0.021)$ were detected in patients older than five years of age (Table S2). The length of hospitalization for patients infected with each pathogen was not significantly different $(P>0.05)$. Pathogens that were associated with dry and cool seasons included rotavirus, followed by norovirus GII, whereas those associated with the other seasons (hot or rainy) included EAEC, Shigella/EIEC, and Plesiomonas (Table S3).

\section{Discussion}

In this study, we investigated the etiological agents of acute diarrhea in patients with severe symptoms by quantitatively detecting a broad range of known enteropathogens. Our highly sensitive molecular assays, as well as bacteriological culture methods, detected high rates of asymptomatic cases and mixed infections in the study population. As the pathogen quantities in stool specimens from case and control subjects estimated using qPCR assays were different (Fig. S1), we set cut-off values of Cq for the specific diagnosis of patients and then predicted the causative pathogens of acute diarrhea. This considerably improved the assay and allowed differentiation between symptomatic and asymptomatic carriage; it also assisted in specifying disease-associated pathogens in the patients. Most illnesses were caused by rotavirus, followed by norovirus GII, Shigella/EIEC, and Campylobacter.

The $\mathrm{Cq}$ values obtained from qPCR assays for case and control samples were compared in each age group or each month (season) to identify trends and patterns of enteropathogen infections. The detection rates of Shigella/ EIEC, Aeromonas, and Plesiomonas were higher in the $\geq 5$ years of age group than in the $<5$ years of age group. In contrast, in addition to rotavirus, norovirus GII, and Campylobacter, ETEC, and sapovirus were likely to be more abundant in younger age groups (Table S2 and Fig. S3). These results might be due to differences in susceptibility to infections and/or lifestyles such as eating habits. Notably, EAEC $(\operatorname{aggR})$ infection was also associated with diarrhea under the cut-off condition and nine of 11 patients were 1 year of age or under. Persistent diarrhea with EAEC is most frequently reported in children aged $\leq 1$ year $^{24}$. Our results thus indicate that infants are more susceptible to EAEC infection than older individuals. Furthermore, regarding asymptomatic infections with norovirus GII, 16 of 17 cases were less than 5 years of age and the remaining one was 6 years old. Infected asymptomatic carriers in young children might be important for the transmission of norovirus infection. Moreover, the seasonal trends of viral infections were clearer than those of bacterial infections (Table S3 and Fig. S2). Rotavirus, 


\begin{tabular}{|c|c|c|c|c|}
\hline \multicolumn{2}{|l|}{ Pathogen } & \multirow[t]{2}{*}{ Genotype or Serogroup } & \multirow{2}{*}{\begin{tabular}{|l|}
$\begin{array}{l}\text { Number of } \\
\text { isolates in cases }\end{array}$ \\
23 \\
\end{tabular}} & \multirow{2}{*}{\begin{tabular}{|l|}
$\begin{array}{l}\text { Number of isolates } \\
\text { in controls }\end{array}$ \\
18 \\
\end{tabular}} \\
\hline Aeromonas & & & & \\
\hline & A. caviae & & 8 & 10 \\
\hline & A. hydrophila & & 4 & 0 \\
\hline & A. sobria & & 11 & 7 \\
\hline & A. veronii & & 0 & 1 \\
\hline \multirow[t]{4}{*}{ Campylobacter } & & & 11 & 2 \\
\hline & C. coli & & 1 & 0 \\
\hline & C. fetus & & 1 & 0 \\
\hline & C. jejuni & & 9 & 2 \\
\hline \multirow[t]{46}{*}{ Escherichia } & E. coli & & 139 & 167 \\
\hline & EAEC & & 55 & 69 \\
\hline & & uidA, aafII, ast $A$ & 0 & 1 \\
\hline & & uidA, ast A, pCVD432 & 3 & 8 \\
\hline & & uidA, astA, pCVD432, pic & 3 & 0 \\
\hline & & uidA, astA, pic & 2 & 2 \\
\hline & & uidA, pic & 8 & 12 \\
\hline & & uidA, pCVD432, pic & 4 & 2 \\
\hline & & uidA, pCVD432 & 10 & 20 \\
\hline & & ast $A, \mathrm{pCVD} 432$ & 0 & 2 \\
\hline & & pic & 2 & 2 \\
\hline & & uidA, aggR & 3 & 3 \\
\hline & & uidA, aggR, ast A & 1 & 1 \\
\hline & & uidA, aggR, ast $A, \mathrm{pCVD} 432$ & 0 & 1 \\
\hline & & $\begin{array}{l}\text { uidA, aggR, ast } A, \text { pCVD432, } \\
\text { pic }\end{array}$ & 2 & 5 \\
\hline & & uidA, aggR, pCVD432 & 7 & 2 \\
\hline & & uidA, aggR, pCVD432, pic & 9 & 4 \\
\hline & & uidA, aggR, pic & 1 & 2 \\
\hline & & aggR, pic, pCVD432 & 0 & 1 \\
\hline & & aggR, pCVD432 & 0 & 1 \\
\hline & EPEC & & 39 & 42 \\
\hline & & uidA, eae & 32 & 24 \\
\hline & & uidA, eae, ast $A$ & 1 & 3 \\
\hline & & uidA, eae, bfpA & 1 & 1 \\
\hline & & uidA, eae, bfpB & 1 & 5 \\
\hline & & uidA, $b f p B$ & 0 & 2 \\
\hline & & uidA, bfpB, ast $A$ & 0 & 1 \\
\hline & & eae & 4 & 6 \\
\hline & ETEC & & 14 & 9 \\
\hline & & uidA, elt & 3 & 1 \\
\hline & & uidA, elt, astA & 4 & 2 \\
\hline & & uidA, elt, est-p & 1 & 0 \\
\hline & & uidA, elt, est-p, ast $A$ & 0 & 1 \\
\hline & & uidA, est-h & 1 & 1 \\
\hline & & uidA, est-h, ast $A$ & 0 & 1 \\
\hline & & uidA, est-h, ast $A$, bfp $A$ & 0 & 1 \\
\hline & & uidA, est-p & 2 & 1 \\
\hline & & uidA, est-p, ast $A$ & 2 & 0 \\
\hline & & elt, est-p & 1 & 0 \\
\hline & & est- $h$, ast $A, b f p A$ & 0 & 1 \\
\hline & STEC & uidA, stxl & 0 & $2^{*}$ \\
\hline & EIEC & & 3 & 3 \\
\hline & & uidA, ipaH, virF & 3 & 1 \\
\hline & & uidA, invE, ast $A$ & 0 & 1 \\
\hline & & $i n v E$ & 0 & 1 \\
\hline & DAEC & & 1 & 5 \\
\hline Continued & & & & \\
\hline
\end{tabular}




\begin{tabular}{|c|c|c|c|c|}
\hline \multicolumn{2}{|l|}{ Pathogen } & \multirow{2}{*}{$\begin{array}{l}\text { Genotype or Serogroup } \\
\text { uidA, daaE }\end{array}$} & \multirow{2}{*}{\begin{tabular}{|l|}
$\begin{array}{l}\text { Number of } \\
\text { isolates in cases }\end{array}$ \\
1 \\
\end{tabular}} & \multirow{2}{*}{\begin{tabular}{|l|}
$\begin{array}{l}\text { Number of isolates } \\
\text { in controls }\end{array}$ \\
4 \\
\end{tabular}} \\
\hline & & & & \\
\hline & & uidA, daaE, ast $A$ & 0 & 1 \\
\hline & Other & & 27 & 37 \\
\hline & & uidA, astA & 27 & 36 \\
\hline & & astA & 0 & 1 \\
\hline \multirow[t]{4}{*}{ Shigella } & & & 11 & 1 \\
\hline & S. boydii & & 0 & 1 \\
\hline & S. flexneri & & 3 & 0 \\
\hline & S. sonnei & & 8 & 0 \\
\hline \multirow[t]{9}{*}{ Salmonella } & S. enterica & & 33 & 33 \\
\hline & & $\mathrm{O} 4$ & 20 & 9 \\
\hline & & O7 & 4 & 10 \\
\hline & & $\mathrm{O} 8$ & 1 & 4 \\
\hline & & O9 & 3 & 2 \\
\hline & & $\mathrm{O} 3,10$ & 3 & 2 \\
\hline & & $\mathrm{O} 13$ & 2 & 1 \\
\hline & & $\mathrm{O} 35$ & 0 & 1 \\
\hline & & Other O-antigen groups & 0 & 4 \\
\hline Plesiomonas & P. shigelloides & & 13 & 9 \\
\hline \multirow[t]{3}{*}{ Vibrio } & & & 4 & 0 \\
\hline & V. fluvialis & & 1 & 0 \\
\hline & V.parahaemolyticus & & 3 & 0 \\
\hline
\end{tabular}

Table 2. Bacterial pathogens isolated from cases and controls and their features. *Production of verotoxin-1 was confirmed by reverse passive latex agglutination, using the VTEC-RPLA kit (Denka Seiken, Japan).

\begin{tabular}{|l|l|l|}
\hline Number of pathogen & Diarrhea & Control \\
\hline 0 & $200(54.1 \%)$ & $201(54.3 \%)$ \\
\hline 1 & $\begin{array}{l}122 \\
(33.0 \%)\end{array}$ & $\begin{array}{l}122 \\
(33.0 \%)\end{array}$ \\
\hline 2 & 33 & 35 \\
$(8.9 \%)$ & $9.5 \%)$ \\
\hline 3 & $\begin{array}{l}14 \\
(3.8 \%)\end{array}$ & $\begin{array}{l}11 \\
(3.0 \%)\end{array}$ \\
\hline 4 & $\begin{array}{l}0 \\
(0.3 \%)\end{array}$ & $(0 \%)$ \\
\hline 5 & $\begin{array}{l}0 \\
(0 \%)\end{array}$ & $(0.3 \%)$ \\
\hline Total & $\begin{array}{l}370 \\
(100 \%)\end{array}$ & $\begin{array}{l}370 \\
(100 \%)\end{array}$ \\
\hline
\end{tabular}

Table 3. Number of bacterial pathogens isolated per subject.

norovirus, and astrovirus were more likely to be abundant in the cool and dry season (November to February), whereas Shigella/EIEC, EAEC (aggR), and Plesiomonas were likely to be abundant in other seasons (rainy and/or hot). This approach can be useful for tracking the distribution and emergence of subclinically-infected persons, as well as diarrheal patients.

Next, we focused on the clinical symptoms of patients who were infected with the detected causative pathogens (Table S1). The patients infected with rotavirus presented with fever and mild to severe diarrhea. Vomiting was more common in rotavirus- and norovirus-infected patients than in all diarrhea patients; however, abdominal pain was less frequent in norovirus-infected patients $(P=0.014)$ than in rotavirus-infected patients $(P=0.139)$. In addition, rates of nausea in rotavirus- and norovirus-infected patients were 18/77 (23.4\%) and 3/29 (10.3\%), respectively. This might indicate a characteristic symptom of norovirus infection, that is, the sudden onset of vomiting. Shigella/EIEC, Aeromonas, Salmonella, and Plesiomonas infections were associated with abdominal pain. In patients positive for Shigella/EIEC, the number of fecal leucocytes (WBCs) and the appearance of erythrocytes (RBCs) in stool were significantly higher, which is one of the known characteristics of Shigellosis ${ }^{25}$. Clinical symptoms deduced from the qPCR results and patient data thus appear to correspond with the generally known symptoms caused by rotavirus, norovirus, or Shigella.

Campylobacter was also detected at significantly higher levels in diarrhea cases using qPCR and culture methods. This genus was likely to cause fever and unlikely to cause high frequent defecation in children. The role of Aeromonas as an etiological agent of acute diarrhea has been controversial ${ }^{26,27}$. Previously, in a challenge study, 


\begin{tabular}{|c|c|c|c|c|}
\hline \multirow[b]{2}{*}{ Selected agents } & \multirow{2}{*}{$\begin{array}{l}\text { Single infection } \\
(\%)\end{array}$} & \multicolumn{2}{|l|}{ Mixed infection } & \multirow[b]{2}{*}{ Total } \\
\hline & & with one pathogen (cases) & with two pathogens (cases) & \\
\hline \multirow{2}{*}{ Rotavirus } & 70 & 8 & 2 & 80 \\
\hline & $(87.5)$ & ast $A^{*}$ (3), Camp (2), NorII (2), Astro (1) & aggR \& astA (1), Camp \& Sal (1) & \\
\hline \multirow[t]{2}{*}{ Norovirus GII } & 20 & 8 & 1 & 29 \\
\hline & $(69.0)$ & Camp (3), Astro (2), Rota (2), Sapo (1) & aggR \& ast A (1) & \\
\hline \multirow[t]{2}{*}{ Shigella/EIEC } & 17 & 4 & 0 & 21 \\
\hline & $(81.0)$ & Sapo (1), Ples (1), astA (1), elt (1) & & \\
\hline \multirow[t]{2}{*}{ Campylobacter } & 10 & 7 & 1 & 18 \\
\hline & $(55.6)$ & NorII (3), Rota (2), Sapo (1), aggR (1) & Rota \& Sal (1) & \\
\hline \multirow[t]{2}{*}{ EAEC $(\operatorname{aggR})$} & 6 & 3 & 2 & 11 \\
\hline & $(54.5)$ & ast A (1), Camp (1), elt (1) & ast $A$ \& Rota (1), astA \& NorII (1) & \\
\hline \multirow[t]{2}{*}{ EAEC (astA) } & 2 & 6 & 2 & 10 \\
\hline & $(20.0)$ & Rota (3), $\operatorname{aggR}(1)$, Ples (1), Shig (1) & Rota \& $\operatorname{aggR}(1)$, NorII \& $\operatorname{aggR}(1)$ & \\
\hline \multirow[t]{2}{*}{ Aeromonas } & 8 & 1 & 0 & 9 \\
\hline & $(88.9)$ & Ples (1) & & \\
\hline \multirow[t]{2}{*}{ Sapovirus } & 4 & 5 & 0 & 9 \\
\hline & $(44.4)$ & Shig (2), Camp (1), Astro (1), NorII (1) & & \\
\hline \multirow[t]{2}{*}{ Astrovirus } & 4 & 4 & 0 & 8 \\
\hline & $(50.0)$ & NorII (2), Rota (1), Sapo (1) & & \\
\hline \multirow[t]{2}{*}{ Salmonella } & 5 & 0 & 2 & 7 \\
\hline & $(71.4)$ & & Rota \& Camp (1), Ples \& elt (1) & \\
\hline \multirow[t]{2}{*}{ Plesiomonas } & 3 & 2 & 1 & 6 \\
\hline & $(50.0)$ & Aero (1), astA (1) & elt \& Sal (1) & \\
\hline \multirow[t]{2}{*}{$\operatorname{ETEC}($ elt $)$} & 3 & 2 & 1 & 6 \\
\hline & $(50.0)$ & $\operatorname{aggR}(1)$, Shig (1) & Ples \& Sal (1) & \\
\hline
\end{tabular}

Table 4. Number of cases and combinations of causative agents estimated among 181 patients. *astA; EAEC (astA), Camp; Campylobacter, Astro; astrovirus, aggR; EAEC (aggR), NorII; norovirus GII, Rota; rotavirus, Sapo; sapovirus, Ples; Plesiomonas, Shig; Shigella/EIEC, Aero; Aeromonas, Sal; Salmonella, elt; ETEC (elt).

diarrhea was demonstrated in only two of 57 human volunteers, with doses ranging from $10^{4}$ to $10^{10} \mathrm{CFU}^{26}$. In this study, many subclinical Aeromonas infections were recognized; however, such infections were associated with diarrhea in the cutoff condition and were accompanied by abdominal pain, nausea, and a high frequency of defecation. Thus, our analysis underscores the risk of illness caused by Aeromonas.

EAEC infection was not associated with any obvious characteristic clinical symptom. Investigations focusing on children (aged $\leq 1$ year) who are probably highly susceptible to infection can be beneficial to understand this pathogen. Two genes, ast $A$ and $\operatorname{aggR}$, were used in our qPCR panel assay to detect EAEC; however, ast $A$ was not always detected together with aggR. astA encodes EAST1 (EAEC heat-stable enterotoxin), which shares the functional properties of the enterotoxin (STa) secreted by ETEC ${ }^{24}$, whereas $a g g R$ is known as a transcriptional regulator (a key virulence regulator) and an important marker for virulent EAEC ${ }^{28}$. ast A was detected not only in some isolates of EAEC, but also in isolates of EPEC, ETEC, EIEC, DAEC (Diffusely adherent E. coli), and non-categorized diarrheagenic $E$. coli (Table 2). Furthermore, single infection with astA-carrying agents was detected in only two of 10 cases (Table 4). These results imply that the presence of ast $A$ is not sufficient to conclusively identify the causative agents of diarrhea. Further, evaluation of the pathogenicity of these suspected pathogens will also be important.

This study has certain limitations. We could not determine the causative agents in approximately $50 \%$ of cases, although many enteropathogens were detected. Our estimates of causative agents were based on differences in pathogen loads between patient and control stool samples. In reality, the association between pathogen quantity and disease is host-pathogen-specific. In addition, pathogen quantities change in each stage of clinical manifestation $^{8,29}$. These issues should be addressed in future studies to accurately predict etiological agents of disease. Further, the lower detection rates of certain enteropathogens such as Vibrio, norovirus GI, and STEC were not sufficient to interpret the qPCR results in this study. Further, the lack of consideration of matching pairs in the statistical analysis is also a limitation.

In a recent report, travelers' diarrhea in foreign visitors to Thailand was investigated using a TaqMan array card assay ${ }^{30}$. One hundred and seventy-three cases from the in-patient or out-patient department in a private hospital and 165 non-diarrheal subjects were enrolled in that study. The results of that study were in agreement with our results regarding the high detection rates of Campylobacter, EAEC, EPEC, and norovirus GII, and the extremely low detection rates of protozoa including Cryptosporidium, Entamoeba histolytica, and Giardia. In contrast, rotavirus, adenovirus, and astrovirus were rarely detected in their study. The low detection rate of rotavirus might be due to the age of their enrolled patients, who were more than 18 years of age. Accordingly, massive surveillance using several approaches will assist in identifying a panel of microbial pathogens associated with acute diarrhea. 
In conclusion, the causative agents of acute diarrhea were predicted using a multiplex qPCR panel assay with patient-specific cut-off values, and the characteristic clinical symptoms caused by each enteropathogen were partially revealed. This approach can provide new insights into infectious diarrheal diseases. Data accumulation, validation of the results, and the re-design/adjustment for known or unknown target enteropathogens in a multiplex quantitative assay could be critical to establish superior diagnostic procedures.

\begin{abstract}
Methods
Specimen collection. Stool specimens were collected from patients hospitalized with acute diarrhea from eight Thai government hospitals nationwide during April 2016 to March 2018 (Table S4). Of all cases, 353 (95.4\%) were patients with abnormally loose stools more than three times within $24 \mathrm{~h}$ and a diarrhea-free time of at least 7 days prior to the collection of stool specimens. The remaining $4.6 \%$ cases were associated with less-frequent defecation. Eligible cases were excluded from the study if they had known immunodeficiency or chronic causes of diarrheal symptoms, or if stool volume was too small $(<3 \mathrm{~g})$ for the experiments. Stool specimens were collected from patients prior to starting the antibiotic treatment in the hospitals or immediately after antibiotic treatment $(<1 \mathrm{~h})$. For each diarrhea case, one healthy volunteer who had no history of diarrhea for at least 30 days before enrolment and who was a match with the case regarding age (mostly within 3 years of the age of the case) and area of residence (living in at least the same province) was selected among the residents or visitors to the hospital by public health officers, coordinators, or nurses. Stool specimens collected in a sterile container were first stored at $4-10^{\circ} \mathrm{C}$ at each site and shipped overnight on ice packs to the central reference laboratory of the National Institute of Health (NIH) of Thailand.
\end{abstract}

Culture methods. Stool samples collected from diarrheal and non-diarrheal control subjects were cultured as part of routine work - for example, direct plating/selective enrichment, isolation, and identification by biochemical tests according to the procedure of the NIH of Thailand. The viable enteric bacteria including Salmonella spp., Shigella spp., Vibrio spp., Aeromonas spp., P. shigelloides, E. coli, and Campylobacter spp. were isolated. Some isolates of enteropathogens were further confirmed or characterized by PCR and serotyping. Five colonies of $E$. coli on MacConkey agar, sorbitol MacConkey agar, Salmonella-Shigella agar, or xylose lysine deoxycholate agar were examined by multiplex-PCR assays ${ }^{31-34}$ for the detection and differentiation of pathogenic E. coli. For the isolation of Campylobacter spp., 1-2 g of stool was enriched in $2 \mathrm{ml}$ Preston broth at $37^{\circ} \mathrm{C}$ for $3-4 \mathrm{~h}$ before dropping stool suspensions on modified charcoal, cefoperazone, deoxycholate agar (mCCDA) and incubating them at $37^{\circ} \mathrm{C}$ for $48 \mathrm{~h}$ in anaerobic jars under microaerophilic conditions (Anaero Pack-MicroAero, Mitsubishi Gas Chemical, Japan).

Quantitative PCR assay. We used TaqMan real-time PCR-based assays for the simultaneous detection of enteropathogens, namely astrovirus (ORF1a), sapovirus (polymerase/capsid junction), adenovirus (hexon), norovirus GI (RdRp/capsid junction), norovirus GII (ORF1-ORF2), rotavirus group A (NSP3), Cryptosporidium spp. (COWP), Giardia lamblia (ITS1), E. histolytica (18S rRNA), P. shigelloides (hugA), Campylobacter spp. (gyrB), Vibrio spp. (toxR), Salmonella spp. (invA), Aeromonas spp. (aerA), Yersinia spp. (lysP), Shigella/EIEC (ipaH), EPEC (eae), ETEC (elt, est-h, est-p), EAEC (aggR, astA), and Shiga toxin-producing E. coli (STEC) (stx1, stx2), following the detection procedure described previously ${ }^{22}$. Several of the defining markers of E. coli pathotypes are proven virulence determinants of the respective pathotype, but for EAEC, the essential virulence determinant has not been proven ${ }^{35}$.

Total RNA and DNA were extracted from individual stool samples using QIAamp viral RNA and QIAamp fast DNA stool mini kits (cat\#52906 and \#51604, Qiagen, USA), respectively, either manually or on a robotic workstation for the automated purification of nucleic acids. For viral RNA/DNA preparation, whole stool (200 mg) was suspended in $1.8 \mathrm{ml}$ saline, which was centrifuged at $4,000 \times g$ for $20 \mathrm{~min}$ and the supernatant containing viral particles was separated. For bacterial and parasitic DNA preparation, diarrheal stool samples were centrifuged at $15,000 \times g$ for $1 \mathrm{~min}$ to obtain a stool pellet (wet weight, $200 \mathrm{mg}$ ). qPCR assays were performed using an Applied Biosystems 7500 Fast real-time PCR system (Foster City, CA, USA). Target genes were amplified using the QuantiFast Pathogen RT-PCR and PCR kits, respectively. Herein, pure water was used as an extraction control to monitor DNA cross-contamination and for environmental contaminants during extraction. For preventing laboratory contamination, preparation of the PCR master mix, extraction of DNA/RNA from stool specimens, addition of DNA/RNA templates to the master mix, and qPCR reactions were conducted in separate areas/rooms in our laboratory. Moreover, each batch of the master mix for real-time PCR reaction was always tested to monitor contamination, along with the test samples.

qPCR data were interpreted according to the following criteria: (i) quantification cycle (Cq) value, defined as the number of PCR cycles where the fluorescent signal exceeded the detection threshold, which was fixed at 0.2 , 0.3 , and 0.5 relative fluorescence units for viral, parasitic, and bacterial targets, respectively; (ii) greater than or equal to a Cq value of 41 was considered as negative for all pathogens in this study; (iii) signals from the internal control (IC) RNA and IC DNA had Cq values of $31 \pm 3$ and $32 \pm 3$, respectively; (iv) positive and no template controls (NTC) were used and verified for validity in every qPCR run ${ }^{22}$.

Statistical analysis. We reported the number of detections and their percentage for each pathogen by case and control groups. We also estimated the ORs and performed Fisher's exact tests. The complex infections were counted and reported as proportions. For each pathogen, Cq values of qPCR-positive samples were presented as median, interquartile range (IQR), and total range. ROC analyses were conducted to detect the optimal cut-off value. The cut-off value with the largest OR, which satisfied more than $95 \%$ specificity, was considered optimal. In all procedures, we calculated the OR based on the contingency table by adding a 0.5 correction value to all cells 
(Haldane-Anscombe 1/2 correction) to address zero- cell in the two-by-two contingency table for case/control and positive/negative. The relationships between case (control) and positive or negative controls based on the calculated cut-off value were reported as ORs and $P$-values, as determined using the Fisher's exact test. All statistical analyses were performed using R (The R Foundation for Statistical Computing, ver. 3.5.1) and all tests were two-tailed. $P$-values $<0.05$ were considered statistically significant.

Ethical statements. The clinical protocol was approved by the Ethical Review Committee for Research in Human Subjects, Ministry of Public Health, Thailand (reference no. 44/2558), Institutional Review Board BIDI (S023q/58), Prapokklao Hospital (CTIREC044), Sunprasitthiprasong Hospital (063/2559), Chiang Rai Prachanukroh Hospital (CR 0032.102/9844), and Institutional Review Board of Maesot General Hospital and Samutsakhon Hospital. Stool specimens were collected from all the volunteers who provided written informed consent. The methods were carried out in accordance with the approved guidelines.

Received: 29 August 2019; Accepted: 13 February 2020;

Published online: 04 March 2020

\section{References}

1. Brown, K. H. Diarrhea and malnutrition. J. Nutr. 133, 328s-332s (2003).

2. Petri, W. A. Jr. et al. Enteric infections, diarrhea, and their impact on function and development. J. Clin. Invest. 118, 1277-1290 (2008).

3. Ferdous, F. et al. Severity of diarrhea and malnutrition among under five-year-old children in rural Bangladesh. Am. J. Trop. Med. Hyg. 89, 223-228 (2013).

4. Sweetser, S. Evaluating the patient with diarrhea: a case-based approach. Mayo Clin. Proc. 87, 596-602 (2012).

5. Fischer Walker, C. L., Sack, D. \& Black, R. E. Etiology of diarrhea in older children, adolescents and adults: a systematic review. PLoS Negl. Trop. Dis. 4, e768 (2010).

6. Operario, D. J. \& Houpt, E. Defining the causes of diarrhea: novel approaches. Curr. Opin. Infect. Dis. 24, 464-471 (2011).

7. Hodges, K. \& Gill, R. Infectious diarrhea: Cellular and molecular mechanisms. Gut Microbes 1, 4-21 (2010).

8. Platts-Mills, J. A., Liu, J. \& Houpt, E. R. New concepts in diagnostics for infectious diarrhea. Mucosal Immunol. 6, 876-885 (2013).

9. Horwood, P. F. et al. A high burden of asymptomatic gastrointestinal infections in traditional communities in Papua New Guinea. Am. J. Trop. Med. Hyg. 97, 1872-1875 (2017).

10. Eibach, D. et al. Application of a multiplex PCR assay for the detection of gastrointestinal pathogens in a rural African setting. BMC Infect. Dis. 16, 150 (2016).

11. Liu, J. et al. Development and assessment of molecular diagnostic tests for 15 enteropathogens causing childhood diarrhoea: a multicentre study. Lancet Infect. Dis. 14, 716-724 (2014).

12. Taniuchi, M. et al. Etiology of diarrhea in Bangladeshi infants in the first year of life analyzed using molecular methods. J. Infect. Dis. 208, 1794-1802 (2013).

13. Phillips, G. et al. Diagnosing norovirus-associated infectious intestinal disease using viral load. BMC Infect. Dis. 9, 63 (2009).

14. Phillips, G. et al. Diagnosing rotavirus A associated IID: Using ELISA to identify a cut-off for real time RT-PCR. J. Clin. Virol. 44, 242-245 (2009).

15. Bolotin, S. et al. Correlation of real time PCR cycle threshold cut-off with Bordetella pertussis clinical severity. PloS One 10, e0133209 (2015).

16. Jansen, R. R. et al. Frequent detection of respiratory viruses without symptoms: toward defining clinically relevant cutoff values. J. Clin. Microbio.l 49, 2631-2636 (2011).

17. Kang, G. et al. Quantitation of group A rotavirus by real-time reverse-transcription-polymerase chain reaction: correlation with clinical severity in children in South India. J. Med. Virol. 73, 118-122 (2004).

18. Barletta, F. et al. Quantitative real-time polymerase chain reaction for enteropathogenic Escherichia coli: a tool for investigation of asymptomatic versus symptomatic infections. Clin. Infect. Dis. 53, 1223-1229 (2011).

19. Elfving, K. et al. Real-time PCR threshold cycle cutoffs help to identify agents causing acute childhood diarrhea in Zanzibar. J. Clin. Microbiol. 52, 916-923 (2014).

20. Li, L. L. et al. Aetiology of diarrhoeal disease and evaluation of viral-bacterial coinfection in children under 5 years old in China: a matched case-control study. Clin. Microbiol. Infect. 22, 381.e389-381.e316 (2016).

21. Liu, J. et al. Use of quantitative molecular diagnostic methods to identify causes of diarrhoea in children: a reanalysis of the GEMS case-control study. Lancet (London, England) 388, 1291-1301 (2016).

22. Wongboot, W., Okada, K., Chantaroj, S., Kamjumphol, W. \& Hamada, S. Simultaneous detection and quantification of 19 diarrhearelated pathogens with a quantitative real-time PCR panel assay. J. Microbiol. Methods. 151, 76-82 (2018).

23. van Maarseveen, N. M., Wessels, E., de Brouwer, C. S., Vossen, A. C. \& Claas, E. C. Diagnosis of viral gastroenteritis by simultaneous detection of Adenovirus group F, Astrovirus, Rotavirus group A, Norovirus genogroups I and II, and Sapovirus in two internally controlled multiplex real-time PCR assays. J. Clin. Virol. 49, 205-210 (2010).

24. Hebbelstrup Jensen, B., Olsen, K. E., Struve, C., Krogfelt, K. A. \& Petersen, A. M. Epidemiology and clinical manifestations of enteroaggregative Escherichia coli. Clin. Microbiol. Rev. 27, 614-630 (2014).

25. Hossain, M. A. \& Albert, M. J. Effect of duration of diarrhoea and predictive values of stool leucocytes and red blood cells in the isolation of different serogroups or serotypes of Shigella. Trans. R. Soc. Trop. Med. Hyg. 85, 664-666 (1991).

26. Morgan, D. R., Johnson, P. C., DuPont, H. L., Satterwhite, T. K. \& Wood, L. V. Lack of correlation between known virulence properties of Aeromonas hydrophila and enteropathogenicity for humans. Infect. Immun. 50, 62-65 (1985).

27. Teunis, P. \& Figueras, M. J. Reassessment of the enteropathogenicity of mesophilic Aeromonas species. Front. Microbiol. 7, 1395 (2016).

28. Sarantuya, J. et al. Typical enteroaggregative Escherichia coli is the most prevalent pathotype among E. coli strains causing diarrhea in Mongolian children. J. Clin. Microbiol. 42, 133-139 (2004).

29. Fredricks, D. N. \& Relman, D. A. Sequence-based identification of microbial pathogens: a reconsideration of Koch's postulates. Clin. Microbiol. Rev. 9, 18-33 (1996).

30. Lertsethtakarn, P. et al. Travelers' Diarrhea in Thailand: A quantitative analysis using TaqMan(R) array card. Clin. Infect. Dis. 67, 120-127 (2018).

31. Muller, D. et al. Identification of unconventional intestinal pathogenic Escherichia coli isolates expressing intermediate virulence factor profiles by using a novel single-step multiplex PCR. Appl. Environ. Microbiol. 73, 3380-3390 (2007).

32. Sjoling, A., Sadeghipoorjahromi, L., Novak, D. \& Tobias, J. Detection of major diarrheagenic bacterial pathogens by multiplex PCR panels. Microbiol. Res. 172, 34-40 (2015). 
33. Chandra, M., Cheng, P., Rondeau, G., Porwollik, S. \& McClelland, M. A single step multiplex PCR for identification of six diarrheagenic E. coli pathotypes and Salmonella. Int. J. Med. Microbiol. 303, 210-216 (2013).

34. Gunzburg, S. T., Tornieporth, N. G. \& Riley, L. W. Identification of enteropathogenic Escherichia coli by PCR-based detection of the bundle-forming pilus gene. J. Clin. Microbiol. 33, 1375-1377 (1995).

35. Robins-Browne, R. M. et al. Are Escherichia coli pathotypes still relevant in the era of whole-genome sequencing? Front. Cell Infect Microbiol. 18, 141 (2016).

\section{Acknowledgements}

The authors would like to thank all the volunteers and the health care professionals who participated in this study. This work was supported by the Japan Initiative for Global Research Network on Infectious Diseases (J-GRID) of the Ministry of Education, Culture, Sports, Science \& Technology of Japan (MEXT), Japan Agency for Medical Research and Development (AMED) under grant number JP18fm0108003, and the Department of Medical Sciences (DMSc), the Ministry of Public Health, Thailand.

\section{Author contributions}

K.O., W.W. and S.H. conceived the study. K.O., W.W. and W.K. designed the protocol. P.K., N.C., W.M., N.A., P.K., P.U., C.T., S.K., L.J., C.J., S.N. and W.S. coordinated specimen collection with hospital staff and patients and clinical data analysis at the site. S.C., P.W., W.W., W.K., N.S., T.W. and P.A.O. performed bacterial culture and characterization of isolates. W.W., W.K. and N.S. performed real-time PCR and data analysis. S.K., W.W. and K.O. performed the statistical analysis. K.O. and S.H. were responsible for the overall design and writing. All authors reviewed the draft and approved the decision to submit for publication.

\section{Competing interests}

The authors declare no competing interests.

\section{Additional information}

Supplementary information is available for this paper at https://doi.org/10.1038/s41598-020-60711-1.

Correspondence and requests for materials should be addressed to K.O.

Reprints and permissions information is available at www.nature.com/reprints.

Publisher's note Springer Nature remains neutral with regard to jurisdictional claims in published maps and institutional affiliations.

(1) Open Access This article is licensed under a Creative Commons Attribution 4.0 International

License, which permits use, sharing, adaptation, distribution and reproduction in any medium or format, as long as you give appropriate credit to the original author(s) and the source, provide a link to the Creative Commons license, and indicate if changes were made. The images or other third party material in this article are included in the article's Creative Commons license, unless indicated otherwise in a credit line to the material. If material is not included in the article's Creative Commons license and your intended use is not permitted by statutory regulation or exceeds the permitted use, you will need to obtain permission directly from the copyright holder. To view a copy of this license, visit http://creativecommons.org/licenses/by/4.0/.

(c) The Author(s) 2020 